\title{
Pediatric Extensor Pollicis Longus Tendon Rupture after Nondisplaced Distal Radius Fracture: A Literature Review
}

\author{
Phong Truong $^{1 *}$, Kristina Kuklova ${ }^{2}$, Abby Halpern ${ }^{1}$, Mitchell Hunter ${ }^{1}$, Eric Heidemann ${ }^{1}$, Felix Stanziola ${ }^{1}$
}

${ }^{1}$ Department of Orthopedic Surgery, Larkin Community Hospital, South Miami, FL, USA

${ }^{2}$ American University of Antigua College of Medicine, St. Johns, Antigua and Barbuda, USA

Received Date: September 28, 2020; Accepted Date: October 07, 2020; Published Date: October 17, 2020

*Corresponding Author: Phong Truong, Department of Orthopedic Surgery, Larkin Community Hospital, 7000 SW 62nd Ave, Suite 401, South Miami, FL 33143, USA. Tel: +13052847500; Email: Ptruong@ larkinhospital.com

\section{Abstract}

The purpose of this article is to review the literature related to extensor pollicis longus tendon rupture following nondisplaced distal radius fractures in the pediatric population. This injury is more commonly seen in adults and is relatively rare in children and adolescents. In our literature search, only one case report of pediatric extensor pollicis longus rupture after nondisplaced displaced distal radius fracture is reported. Therefore, it is possible that anatomic and physiologic differences in pediatric patients may be protective against the physical irritation and ischemia thought to cause this injury in their adult counterparts. More research, including biomechanical studies and vascular studies, ought to be done to evaluate for the physiologic differences in pediatric patients that may account for the decreased risk of extensor pollicis longus rupture after nondisplaced distal radius fractures.

Keywords: Displaced; Distal Radius Fracture; EPL; Extensor Pollicis Longus; Nondisplaced; Pediatrics

\section{Abbreviations}

$\begin{array}{ll}\text { EPL: } & \text { Extensor Pollicis Longus } \\ \text { FPL: } & \text { Flexor Pollicis Longus } \\ \text { EIP: } & \text { Extensor Indicis Proprius }\end{array}$

Introduction

Extensor pollicis longus (EPL) tendon rupture is a complication associated with nondisplaced fractures of the distal radius in adults. In 2013, the estimated frequency of these injuries ranged from $0.07 \%$ to $0.88 \%$ [1], but the more recent 2018 studies reported incidence of $0.4 \%$ to $5 \%$ occurring six weeks after the fracture [2].

The etiology of delayed EPL tendon rupture is still unclear, but accepted belief is that the injury occurs due to a degenerative process secondary to ischemic atrophy, physical irritation, or a combination of the two [3].

The incidence of EPL tendon injuries in the pediatric population is significantly lower compared to the adults, but there is still limited research regarding this topic. Previously written reports suggest that EPL tendon ruptures in kids occur more commonly following displaced fractures or those requiring intramedullary nailing and open reduction, as the implants provide another source of tendon irritation [1].

\section{Methods}

A review of literature was conducted. Using the MEDLINE database and Google Scholar search engine, publications in the English language were queried in regards to extensor pollicis longus tendon rupture in adults and pediatrics. Example search queries included "extensor tendon pollicis tendon rupture pediatrics" and "nondisplaced distal radius extensor tendon rupture". Bibliographies of chosen studies were searched for additional sources. A total of 24 sources were included in the review.

\section{Mechanism of EPL Rupture}

Mechanical irritation plays a role in the rupture of the EPL. Nondisplaced injuries of the distal radius preserve the integrity of the extensor retinaculum, which in turn leads to EPL tendon rupture as it is held against the fracture callus. In displaced fractures, the extensor retinaculum is frayed causing no direct contact of EPL tendon with the fracture site [4]. Repetitive motion while performing a specific type of exercise can also cause irritation and rupture [5]. A case report of a 23-year-old male kickboxer presented with pain and inability to extend the interphalangeal joint of the left thumb for the last 24 hours. He had no history of trauma, but stated that the day before he was doing reverse press ups on the dorsum of his hands with wrists being in hyperflexed position. Very likely that it caused EPL irritation because of its direct pressure on the dorsal tubercle of the radius [5].

Another cause of EPL tendon rupture is thought to be secondary to attrition and ischemic necrosis from hemorrhage and increased pressure within the tendon sheath [6]. In support of this, Engkvist reports that part of the EPL tendon is poorly vascularized, therefore making it susceptible to rupture from increased pressure. A hematoma in the tendon sheath could also interfere with synovial fluid production, depriving the tendon of nutrition, causing further attrition [7]. Zvijac et al finds that EPL tendon may rupture without severe trauma, inflammatory disease, or synovitis; attritional injury may occur around Lister's tubercle [8].

\section{EPL Rupture in Adults}

EPL rupture is a known complication following distal radius fractures. The incidence of EPL tendon rupture in the setting of nondisplaced distal radius fractures is relatively unknown. However, there have been more recent studies showing an incidence of up to $5 \%$, which is higher than previously reported. This percentage of injuries is reported at an average of 6.6 weeks following injury [2]. One complication of a distal radius fracture may involve entrapment of EPL in the 
fracture site which can cause limitation in thumb extension [9]. Another unique injury mechanism is a fracture of Lister's tubercle. Traumatic hyperextension of a patient's wrist can cause compression of the third metacarpal into Lister's tubercle causing a small fracture. Chronic chafing of the EPL on the jagged surface can lead to spontaneous EPL rupture [10].

Volar plate fixation with locked screws is the largely preferred treatment of displaced distal radius fractures that cannot be managed nonoperatively. Although the most common tendon injury seen in volar plating involves the flexor pollicis longus (FPL), extensor tendon injury has also been experienced by a number of surgeons [11]. Proposed reasons for these extensor tendon injuries in volar plating include improper screw measurement leading to screw protrusion into the extensor tendons, and insufficient reduction of the dorsal roof fragments of the distal radius. Due to this, the surgeon must determine the optimum screw length carefully and the dorsal roof fragments should be adequately reduced [12].

Although rupture of the EPL tendon is a well-known complication of nondisplaced distal radius fractures, there have been numerous reports of EPL rupture in a variety of cases. These include blunt trauma, stab wounds, in operative cases poor plate positioning causing chafing, wear against the pins of an external fixator, steroid use, and even local steroid injection [13]. Cases of EPL rupture have been reported in relationship to a positive rheumatoid factor even in the absence of clinical, or radiologic findings of rheumatoid arthritis [14]. Even in the absence of the aforementioned pathologic or iatrogenic conditions, EPL rupture may be seen in patients with repetitive wrist motions such as restaurant chefs and farmers who milk cows [15].

There are many etiologies that may be related to the observed injury and a thorough history should be obtained when generating a cause. In the majority of cases the treatment of choice is a transfer of the extensor indicis proprius (EIP) tendon to replace the EPL. This treatment has been proven to have great outcomes and restore satisfactory thumb function in the majority of patients [16].

\section{EPL Rupture in Pediatrics}

In a 2014 review, it was mentioned that there are only nine cases in the English literature regarding delayed EPL tendon rupture in kids, five of which were not reported in detail [17]. One of these case reports mentions a 12-year-old boy presenting to the emergency department after a fall from his bicycle, sustaining a closed distal radius fracture with physeal separation and palmar displacement. Patient underwent surgical treatment followed by wearing a long-arm cast for 6 weeks. Once the cast was removed, the boy had trouble extending his thumb due to the EPL tendon rupture. This was confirmed using a sonogram, no continuity of the tendon was observed as it became invisible closer to the level of the distal radius [18]. Another case involves also a 12-year-old boy with delayed EPL injury, which occurred one month post closed reduction and percutaneous pinning of a markedly volarly displaced fracture of the distal radius [19].

An elastic stable intramedullary nail fixation has been recognized as a suitable method of treatment for diaphyseal fractures in radius and ulna in the pediatric population. It has a high success rate. Due to its minimally invasive nature, this procedure is considered safe as it does not compromise physeal growth, yet it could cause injury to the superficial branch of the radial nerve and rupture of EPL tendon [17]. This observation was tested in a case report of an 11-year-old boy, who sustained an injury to his left forearm during a football game. He was found to have an open fracture of the diaphysis with displacement of the radius and ulna. The patient underwent surgery with internal fixation using elastic intramedullary nails for each forearm bone. Twelve weeks later, at the follow up visit, the boy was pain-free and had a full range of motion at the elbow and wrist. But it was noted that the patient lost active extension of the thumb at the interphalangeal joint, which granted a diagnosis of extensor pollicis longus tendon rupture [20].

In our literature search, only one case report of pediatric EPL rupture after nondisplaced displaced distal radius fracture is found. A 15-year-old boy presented to the emergency department with a nondisplaced Salter-Harris II right distal radius fracture sustained during a soccer game. The patient was treated nonoperatively in cast for five weeks; one month later he was unable to extend his thumb. This prompted surgical exploration, which revealed EPL entrapment in the musculotendinous junction. With the absence of bony irregularity or spicule, or prominent Lister's tubercle, it was assumed that EPL injury in this patient could have been caused by ischemia of the tendon due to vascular compromise [1].

Unlike in adults, pediatric EPL tendon rupture occurs more commonly after displaced fractures. Possible causes include mechanical etiology from a bony spicule versus iatrogenic after open reduction and internal fixation [3]. EPL tendon rupture presents to be an issue post elastic intramedullary nailing of the radius, particularly when the dorsal entry approach is utilized. Awareness should be raised for an increased risk of EPL rupture with this fixation method [21]. In order to avoid direct injury to the tendon using this technique, surgeons are advised to operate by advancing laterally, which is a safer alternative regarding EPL injuries [22]. Another possible cause of delayed EPL rupture is due to the tendon irritation over a protruding nail end following elastic nailing [23].

The majority of distal radius fractures in the pediatric population are treated nonoperatively, unless there are concomitant neurovascular instabilities or soft tissue interposition between fracture fragments prior to the anatomical reduction. In which case, surgical repair becomes part of the treatment differential. A case of a 14-year-old boy provides support to a theory of EPL tendon rupture being caused by its entrapment between the fracture fragments. He presented to the emergency department with left wrist pain after falling from a bicycle. Proper radiographic tests were ordered and demonstrated a displaced irreducible distal radius fracture, so the patient was taken to the operating room for open reduction and volar plate fixation. At the six weeks postoperative follow-up visit, the boy was unable to extend his thumb, requiring an ultrasound to be done. The test showed EPL tendon entrapment near the fracture site. Similar case was reported in 2013 in the youngest known patient in the literature, 9-year-old boy, who presented with a pediatric distal radius fracture and a later complication of EPL tendon injury due to its entrapment in fracture callus [24].

\section{Conclusion}

Pediatric EPL tendon rupture is more commonly seen after displaced fractures, while in adults it tends to occur more commonly in nondisplaced distal radius fractures. The proposed mechanisms for adults sustaining EPL ruptures are related to physical irritation and vascular compromise. In nondisplaced distal radius fractures, the intact extensor mechanism holds the EPL tendon against the fracture site, producing irritation, while hemorrhage in the tendon sheath increases pressure and causes vascular compromise leading to ischemia. Therefore it is possible that the periosteum in children produces less irritation than the cortical bone in adults. Also, physiologic differences in pediatrics may be protective against the ischemia seen in their adult counterparts. More research, including biomechanical studies and vascular studies, ought to be done to evaluate for the physiologic differences in pediatric patients after nondisplaced distal radius fractures that may account for the decreased risk of EPL rupture. 


\section{Author Contribution Statement}

Authors Phong Truong, Kristina Kuklova, Abby Halpern, Mitchell Hunter, Eric Heidemann, and Felix Stanziola contributed to the conception or design of the article; drafted or critically revised the article; approved the final version to be published; and agreed to be accountable for all aspects of the article

\section{Conflicts of Interest}

We declare that we have no conflicts of interest in the authorship or publication of this article.

\section{References}

1. Song D, Evans R, Arneja JS (2013) Delayed extensor pollicis longus tendon rupture following nondisplaced distal radius fracture in a child. Hand (N Y) 8: 242-244. doi:10.1007/s11552-013-9494-9

2. Roth KM, Blazar PE, Earp BE, Han R, Leung A (2012) Incidence of extensor pollicis longus tendon rupture after nondisplaced distal radius fractures. J Hand Surg Am 37: 942-947. doi:10.1016/j.jhsa.2012.02.006

3. Bogart R, Vidlock K (2020) Ruptured Extensor Pollicis Longus Tendon After a Nondisplaced Distal Radius Fracture in a Young Adult Soccer Player. Clin J Sport Med 30: e23-e24. doi:10.1097/JSM.0000000000000708

4. Helal B, Chen SC, Iwegbu G (1982) Rupture of the Extensor Pollicis Longus Tendon in Undisplaced Colles' Type of Fracture. Hand 14: 41-47. doi:10.1016/S0072$968 \mathrm{X}(82) 80038-7$

5. Lloyd TW (1998) Spontaneous rupture of extensor pollicis longus tendon in a kick boxer. Br J Sports Med 32: 178-179. doi:10.1136/bjsm.32.2.178

6. Gelb R (1995) Tendon transfer for rupture of the extensor pollicis longus. Hand Clin 11: 411-422.

7. Engkvist O, Lundborg G (1979) Rupture of the Extensor Pollicis Longus Tendon after Fracture of the Lower End of the Radius-a Clinical and Microangiography Study. Hand 11: 76-86. doi:10.1016/S0072-968X(79)80015-7

8. Zvijac JE, Janecki CJ, Supple KM (1993) Non-traumatic spontaneous rupture of the extensor pollicis longus tendon. Orthopedics 16: 1347-1350. doi:10.3928/01477447-19931201-10

9. Murakami Y, Todani K. Traumatic entrapment of the extensor pollicis longus tendon in Smith's fracture of the radius-Case report. J Hand Surg Am 6: 238-240. doi:10.1016/S0363-5023(81)80076-7

10. Stahl S, Wolff TW (1988) Delayed rupture of the extensor pollicis longus tendon after nonunion of a fracture of the dorsal radial tubercle. J Hand Surg Am 13: 338-341. doi:10.1016/S0363-5023(88)80004-2

11. Monaco NA, Dwyer CL, Ferikes AJ, Lubahn JD (2016) Hand surgeon reporting of tendon rupture following distal radius volar plating. Hand 11: 278-286. doi:10.1177/1558944715620792
12. Zenke Y, Sakai A, Oshige T, Moritani S, Menuki K, et al. (2013) Extensor pollicis longus tendon ruptures after the use of volar locking plates for distal radius fractures. Hand Surg 18: 169-173. doi:10.1142/S0218810413500196

13. Björkman A, Jörgsholm P (2004) Rupture of the extensor pollicis longus tendon: A study of aetiological factors. Scand J Plast Reconstr Surg Hand Surg 38: 32-35. doi:10.1080/02844310310013046

14. Choi JC, Kim WS, Na HY, Lee YS, Song WS, et al. (2011) Spontaneous Rupture of the Extensor Pollicis Longus Tendon in a Tailor. Clin Orthop Surg 3: 167. doi:10.4055/cios.2011.3.2.167

15. Taş S, Balta S, Benlier E (2014) Spontaneous rupture of the extensor pollicis longus tendon due to unusual etiology. Balkan Med J 31: 105-106. doi:10.5152/balkanmedj.2013.9027

16. Noordanus RP, Pot JH, Jacobs PBD, Stevens K (1994) Delayed rupture of the extensor pollicis longus tendon: a retrospective study. Arch Orthop Trauma Surg 113: 164166. doi:10.1007/BF00441626

17. Brooker B, Harris PC, Donnan LT, Graham HK (2014) Rupture of the extensor pollicis longus tendon following dorsal entry flexible nailing of radial shaft fractures in children. J Child Orthop 8: 353-357. doi:10.1007/s11832014-0605-0

18. El-Kazzi W, Schuind F (2005) Extensor pollicis longus entrapment in a paediatric distal radius fracture. J Hand Surg Am 30: 648-649. doi:10.1016/j.jhsb.2005.06.015

19. Kay RM, Khounganian GS, Stevanovic M (2004) Late extensor pollicis longus rupture following displaced distal radius and ulna fractures in a child. J Orthop Trauma 18: 53-56. doi:10.1097/00005131-200401000-00011

20. Kravel T, Sher-Lurie N, Ganel A (2007) Extensor pollicis longus rupture after fixation of radius and ulna fracture with titanium elastic nail (TEN) in a child: A case report. J Trauma - Inj Infect Crit Care. 2007;63(5):1169-1170. doi:10.1097/TA.0b013e31802e3fd9 https://pubmed.ncbi.nlm.nih.gov/17993966/

21. Lee AK, Beck JD, Mirenda WM, Klena JC (2016) Incidence and Risk Factors for Extensor Pollicis Longus Rupture in Elastic Stable Intramedullary Nailing of Pediatric Forearm Shaft Fractures. J Pediatr Orthop. 36: 810-815. doi:10.1097/BPO.0000000000000568

22. Murphy HA, Jain VV, Parikh SN, Wall EJ, Cornwall R, et al. (2019) Extensor Tendon Injury Associated with Dorsal Entry Flexible Nailing of Radial Shaft Fractures in Children: A Report of 5 New Cases and Review of the Literature. J Pediatr Orthop 39: 163-168. doi:10.1097/BPO.0000000000000897

23. Stahl S, Calif E, Eidelman M (2007) Delayed rupture of the extensor pollicis longus tendon following intramedullary nailing of a radial fracture in a child. J Hand Surg 32: 6768. doi:10.1016/j.jhsb.2006.08.017

24. Mansour AA, Watson JT, Martus JE (2013) Displaced Dorsal Metaphyseal Cortex Associated With Delayed Extensor Pollicis Longus Tendon Entrapment in a Pediatric Smith's Fracture. J Surg Orthop Adv 22: 173175. doi: $10.3113 /$ JSOA.2013.0173

Citation: Truong P, Kuklova K, Halpern A, Hunter M, Heidemann E, Stanziola F (2020) Pediatric Extensor Pollicis Longus Tendon Rupture after Nondisplaced Distal Radius Fracture: A Literature Review. Adv Ortho and Sprts Med: AOASM-132. 